\title{
BioéthiqueOnline
}

\section{Art + Bioéthique : expériences interdisciplinaires dans une} galerie émergente

\section{Aïda Lorrain}

Volume 5, 2016

URI : https://id.erudit.org/iderudit/1044289ar

DOI : https://doi.org/10.7202/1044289ar

Aller au sommaire du numéro

Éditeur(s)

BioéthiqueOnline

ISSN

1923-2799 (numérique)

Découvrir la revue

Citer ce compte rendu

Lorrain, A. (2016). Compte rendu de [Art + Bioéthique : expériences interdisciplinaires dans une galerie émergente]. BioéthiqueOnline, 5. https://doi.org/10.7202/1044289ar
Résumé de l'article

Ce compte rendu retrace l'exposition et les principaux évènements qui ont marqué le projet collaboratif Art + Bioéthique. Ceux-ci se sont tenus à la galerie montréalaise Espace Projet du 25 février au 21 mars 2016. 


\section{Art + Bioéthique : expériences interdisciplinaires dans une galerie émergente}

COMPTE RENDU / REVIEW

Aïda Lorrain ${ }^{1}$

Reçu/Received: 1 Apr $2016 \quad$ Publié/Published: 16 Sept 2016

Éditrices/Editors: Lise Lévesque \& Mariana Nunez

2016 Aïda Lorrain, Creative Commons Attribution 4.0 International License

\section{Résumé}

Ce compte rendu retrace l'exposition et les principaux évènements qui ont marqué le projet collaboratif Art + Bioéthique. Ceux-ci se sont tenus à la galerie montréalaise Espace Projet du 25 février au 21 mars 2016.

\section{Mots clés}

exposition, art, bioéthique, évènements, conférences, médiation culturelle et scientifique, galerie, projet interdisciplinaire, collaboration

\section{Summary}

This review retraces the exhibition and the principle events of the collaborative project Art + Bioéthique. These were held at the Montreal gallery Espace Projet from February 25 to March 21, 2016.

\section{Keywords}

exhibition, art, bioethics, events, conferences, cultural and scientific mediation, interdisciplinary project, collaboration
Affiliations des auteurs / Author Affiliations

${ }^{1}$ Artiste et commissaire indépendante, Montréal, Canada

Correspondance / Correspondence

Aïda Lorrain, aida.lorrain@gmail.com

Conflit d'intérêts

Aucun déclaré
Conflicts of Interest

None to declare

\section{Le contexte}

Ce compte rendu retrace les évènements de l'exposition Art + Bioéthique, présentée du 25 février au 21 mars 2016 à la galerie montréalaise Espace Projet. Le lecteur se prêtant au jeu, à défaut de sa présence physique lors de ces évènements, peut ainsi faire l'expérience des œuvres et de leur mise en espace dans la galerie ; (re)vivre les moments forts du programme de conférences et s'imaginer comme participant aux activités de médiation. L'intertextualité du projet Art + Bioéthique peut également être activée, en suivant les hyperliens qui unissent les œuvres aux essais des bioéthiciens publiés dans ce dossier thématique. L'auteure était employée de la galerie durant ce programme : à l'accueil des publics, elle a suivi de près la réception du projet. Ce texte offre ainsi un point de vue complémentaire à la présentation des fondements d'Art + Bioéthique par Vincent Couture, JeanChristophe Bélisle-Pipon et Maude Laliberté [1] ainsi qu'à l'analyse des œuvres sous l'angle du travail des bioéthiciens par les commissaires Catherine Barnabé [2] et Marianne Cloutier [3].

\section{Les œuvres}

Dès la rue, l'œuvre Jour de fête! [4] interpelle les passants, ébahis par l'étrangeté de cette photographie imposante à la composition déroutante. A-t-on bien vu poindre la tête d'un bébé, dans ce théâtre où se mêlent chairs, fluides sanguinolents et blouses médicales? À l'intérieur de l'espace d'exposition, on se retrouve face à une image-choc, représentant un accouchement. La photographie est renversée, c'est donc l'expérience du bébé que l'on subjective plutôt que celle de sa mère en plein travail, délibérément à l'envers. Même le cartel défie les repères. Les détails de réalisation de l'encadrement, les dimensions, mais aussi le poids de l'œuvre et l'année (2016) préfigurent à une liste où s'enchaînent les noms de l'artiste Arkadi Lavoie-Lachapelle, de son collaborateur bioéthicien JeanFrédéric Ménard [5], du crédit photo Isabelle Brabant, de l'encadreur Martin Schop et du soutien 
technique à l'impression Mathieu Jacques, suivis par des remerciements adressés à Espace Projet et à l'équipe invisible. Zèle éthique autour de la fabrication conceptuelle d'un ready-made sans doute, mettant à jour les coulisses de la collaboration.

Non loin de là se trouve une table sur laquelle sont disposées des copies de textes rédigés par les bioéthiciens Jean-Frédéric Ménard [5] ; Dominick Rathwell-Deault [6] ; Annie Carrier [7] ; JonasSébastien Beaudry [8] ; Victoria Doudenkova [9] et Mathieu Noury [10]. On s'aperçoit assez vite de l'influence de ces six scientifiques sur les créations des six artistes exposants avec lesquels ils ont été jumelés - et vice-versa. La combinaison texte et œuvre souligne ainsi la nature interdisciplinaire de cette exposition.

En poursuivant la visite on découvre l'œuvre d'Audrey Kinkead [11], 54 rue DuBalcon, une série de documents encadrés sous-verre présentés au mur avec une enveloppe. Cet ensemble conceptuel invite à suivre la piste de Sciurus carolinensis, mieux connu sous le nom d'écureuil, en parcourant les indices d'un document à l'autre. Le premier document est une capture d'écran tirée de la section " logement à louer » du site de petites annonces Kijiji : un cabanon de balcon avec vue sur le parc, dont la description est accompagnée de photos, semble indiquer un domicile pour rongeurs. Le second document est le facsimilé d'un bail de locataire, adressé à Sciurus carolinensis. On ne sait comment l'artiste s'y est prise pour que l'animal griffonne sa patte à l'endroit indiqué, car le contrat est bel et bien signé! Le troisième document est un portrait de notre écureuil en œil-de-bœuf. Ses yeux censurés protègent son identité ; la photographie a peut-être été tirée à son insu... Que contient cette enveloppe, en dernier lieu, scellée, affranchie et toujours adressée à notre ami l'écureuil? On sait seulement qu'elle est expédiée par Dominick Rathwell-Deault [6], bioéthicienne dont les recherches s'articulent autour du concept de responsabilité morale des médecins vétérinaires en regard de leurs patients animaux.

À gauche, une ouverture dans le mur laisse entrevoir un cagibi, dans lequel se trouve l'installation Nano-body de Stephanie Coleman [12]. En entrant dans cette pièce exigüe, on pénètre dans un univers personnel, à mi-chemin entre le laboratoire humain et le boudoir victorien. L'un des murs est tapissé d'illustrations incrustées de petites ampoules DEL, que l'on allume en appuyant aux endroits indiqués par des empreintes digitales. Ces dessins réalisés à l'encre noire sur du papier blanc détaillent un monde hybride où s'enchevêtrent, tels des jardins luxuriants, des cellules nanoscopiques et des plantes. À partir du coin, des cellules commencent à se détacher de la paroi du mur. Légères et gracieuses, ces formes organiques réalisées avec du fil se déploient dans l'espace, créant ainsi une transition aérienne entre la planéité du dessin et les boîtes de pétri situées en face. Celles-ci, disposées sur neuf tablettes individuelles, contiennent des objets précieux rappelant des formations de vertèbres miniatures et des petits globules couleur corail.

À la sortie de la petite pièce, on découvre sur la droite un cactus en pot déposé sur une tablette. La plante est peinte couleur d'or et ses piquants sont enjolivés de petites fleurs rouges. En s'écartant du mur, trois images de cactus modifiés de la même façon se présentent à côté de la plante. Grace Stokes a simulé un halo scintillant artificiel autour de chacun de ces cactus dans ces images créées par procédé numérique et imprimées sur papier photo. Leur titre commun, PCOS as Cacti [13], indique que ces cactus transformés sont en effet des métaphores visuelles pour les effets secondaires vécus par les femmes atteintes du syndrome des ovaires polykystiques [9]. La quatrième image, une sérigraphie numérotée intitulée Constant Beauty [13], est une représentation anatomique et schématique de l'appareil reproducteur féminin à la différence des ovaires, ici remplacés par des fleurs de prunier mirobolant.

Dans le coin gauche de la galerie, au sein d'un espace adapté à la hauteur d'un fauteuil roulant, on regarde le film «On va tenter un serrage de main au travers de l'Atlantique » [14] sur un écran. Faisant partie de l'œuvre performative et évolutive France-e(s)t-Mael, cette vidéo documente la performance de l'artiste Mael Le Mée à Bordeaux (FR) en pleine démonstration publique dans un 
dispositif digne d'un inventeur fou. L'artiste se déplace dans la ville, assis dans un fauteuil roulant électrique muni d'un bras sur lequel une tablette diffuse en direct l'image et la voix d'une interlocutrice. Une écharpe lie affectueusement le duo, dont les deux têtes apparaissent côte à côte tel un " duo siamois transatlantique ». Un ordinateur portable est déposé sur ses genoux de Le Mée, son bras gauche branché à un boîtier d'électrostimulation grâce à deux paires d'électrodes: l'interlocutrice contrôle les mouvements du bras de Le Mée à l'aide d'impulsions déchargées par les électrodes. Un texte rédigé par l'artiste permet d'en savoir plus : l'interlocutrice est France Geoffroy, pionnière de la danse contemporaine intégrée au Québec. Ensemble, ils ont réalisé la performance inverse lors du vernissage de l'exposition. C'est-à-dire que l'artiste, à partir de son bureau situé à Bordeaux, contrôle les mouvements de mains de France, présente dans la galerie au même moment, elle-même munie du dispositif tablette-électrodes et prête à rencontrer le public. Deux portraits placés côte à côte au-dessus de l'écran témoignent de ces deux performances croisées.

Enfin, sur le mur ouest de la galerie se trouve l'œuvre dyptique de Karine Turcot [15]. Dans chacun des cadres, un léger papier ocre voile une reproduction gravée d'un plan d'ensemble architectural, représentant un hôpital du XVIIle siècle. D'autres fragments polycopiés de gravures illustrant le classicisme et les pratiques médicales de l'époque parsèment les pièces délimitées par le plan. Certains fragments sont munis d'attaches velcro au verso et peuvent être déplacés ou retirés de l'œuvre.

\section{Les activités de médiation}

En parallèle à l'exposition, Art + Bioéthique propose un atelier de création pour adultes, une activité de médiation pour enfants ainsi qu'un programme de conférences à Espace Projet.

À l'occasion du festival noctambule Nuit blanche, en partenariat avec Montréal en Lumières, l'atelier de création Post+humain(e) invite une trentaine de participants de réfléchir aux enjeux éthiques du post-humanisme avec Arkadi Lavoie-Lachapelle et Vincent Couture, puis de réaliser une empreinte de plâtre moulée sur une partie du corps et d'y ajouter des pièces électroniques, afin de visualiser les innovations génétiques et technologiques du corps humain. Les œuvres réalisées par les participants placés en vitrine pour la durée de l'exposition attirent l'œil curieux des passants.

L'artiste Audrey Kinkead organise une matinée d'excursion pour les enfants dans les environs de la galerie, au parc Jarry, avec le Laboratoire d'observation du vivant (L.O.V.). Munis de macarons et de fiches d'observations, les enfants portent attention aux environnements de vie des animaux en ville.

\section{Le programme de conférences}

Le format des conférences est varié et libre. Chaque semaine à Espace Projet, deux conférenciers un artiste et un chercheur de l'exposition Art + Bioéthique - présentent un aspect important de leur recherche. La première occurrence est une présentation d'Annie Carrier [7] portant sur l'ambigüité éthique relative au transfert des connaissances scientifiques s'appuyant sur les principes de base de la communication efficace et le rôle du chercheur dans la société. Cette présentation est suivie par un artist talk en anglais donné par Stephanie Coleman à propos de son projet Na-no-body [12] ainsi que de l'influence de cette œuvre sur sa démarche artistique globale. Coleman parle entre autres de la collaboration fructueuse avec son binôme Mathieu Noury, qui a manifesté sa surprise face à l'aspect "low-tech» de son œuvre étant donné son inspiration par une technologie de pointe telle que la nanomédecine [10].

La seconde occurrence est une projection de la conférence préenregistrée de Jonas-Sébastien Beaudry, en anglais, portant sur les réponses affectives négatives véhiculées en société en réaction aux personnes possédant des déficiences intellectuelles ou physiques, ainsi que de l'instrumentalisation des personnes handicapées [12]. Le chercheur n'est pas présent pour 
commenter son intervention, en revanche l'artiste Mael Le Mée, qui a réalisé son œuvre suite à des échanges avec Beaudry, est présent par Skype pour participer à la période de discussion qui suit. La danseuse France Geoffroy est également présente en salle afin de partager son expérience de création avec Le Mée, elle insiste sur le fait que l'œuvre France-e(s)t-Mael ne parle pas de handicap, mais bien d'hybridation des corps [14]. Ensuite, vient le tour de Le Mée de prendre la parole : il situe sa création dans le cadre des études transgenres et dans les théories du post-humanisme, puis élabore sur ce qu'il appelle « la fabrique contemporaine des corps ». Le Mée tranche distinctement le rôle de l'artiste de celui du chercheur bioéthicien : pour lui, l'artiste doit constamment remettre en question les limites du monde, alors que ce n'est pas le cas du bioéthicien.

La troisième occurrence est une "conférence-perf » intitulée Cobayes de la relève, exécutée par Arkadi Lavoie-Lachapelle et Jean-Frédérick Ménard [4-5]. Cette soirée dynamique combine habilement des moments de performance, tels que l'acte cérémonial effectué en retournant à l'endroit l'œuvre Jour de fête, ou une acrobatie réalisée par l'artiste, et des moments de présentation plus standard pendant lesquels l'artiste et le chercheur présentent leurs démarches. Cette conférence est conclue par un geste inattendu : Ménard, qui est avocat, distribue à l'assistance avec un contrat légal, rédigé avec l'aide de Lavoie-Lachapelle, pour la préservation des droits de l'artiste sur toute œuvre cédée, à l'instar du contrat Provjansky (1971)!

La quatrième et dernière occurrence est une conférence donnée par la chercheuse bioéthicienne Victoria Doudenkova [9] et par l'artiste Grace Stokes [13] pendant laquelle est discutée l'interinfluence du sujet de recherche de l'une, les effets secondaires liés au Syndrome des ovaires polykystiques, et la pratique artistique de l'autre. En prenant la parole chacune à leur tour, la présentation se déplace

entre le Canada et l'Angleterre où Stokes réside en ce moment. À travers ce va-et-vient, le duo présente les aléas des projets collaboratifs internationaux.

\section{Conclusion : La collaboration}

Ce compte rendu ne documente que les aspects artistiques, sociaux et éphémères d'Art + Bioéthique. Ce constat d'incomplétude met en valeur la dimension profondément collaborative du projet. Art + Bioéthique est né sous le signe de la collaboration et s'est manifesté à travers des œuvres prenant pour assise des sujets de recherche variés et dont les formes s'avèrent très diversifiées ; des textes sortant du cadre scientifique habituel ; un catalogue d'exposition ; des activités de médiation créées pour des publics différents ; des conférences originales et instructives ; un vernissage performatif, en plus des nombreuses rencontres entre les collaborateurs en phase de préparation de l'exposition. C'est dans tous ces évènements reliés qu'émerge ce laboratoire vivant d'hybridation de l'art et de la bioéthique. Une autre métaphore biologique que l'on pourrait utiliser pour décrire le projet est celle du commensalisme : le cadre artistique a permis la rencontre de recherches interdisciplinaires avec un public réceptif et diversifié, ouvert à l'esprit de l'expérience ; le cadre bioéthique a ouvert des espaces de dialogues et de sens en offrant au public et aux artistes ses thématiques au cœur de sa réflexion sur le devenir de nos sociétés.

\section{Références}

1. Couture V, Bélisle-Pipon JC, Laliberté M. Art + Bioéthique : quand la recherche en bioéthique quitte les murs de l'université. BioéthiqueOnline. 2016;5/16.

2. Barnabé C. Art + Bioéthique : L'art comme médiation. BioéthiqueOnline. 2016;5/29.

3. Cloutier M. Faire dialoguer les cultures : rencontre entre la bioéthique et l'art contemporain. BioéthiqueOnline. 2016;5/30.

4. Lavoie-Lachapelle A. Jour de fête! BioéthiqueOnline. 2016;5/17.

5. Ménard J-F. Cobayes de la relève : l'artiste et le chercheur à la rencontre de l'expérience. BioéthiqueOnline. 2016;5/18.

6. Rathwell-Deault D. L'animal un co-citoyen, et pourquoi pas? BioéthiqueOnline. 2016;5/24. 
7. Carrier A, Contandriopoulos D. Principes de communication et rôle social du chercheur en matière de transfert de connaissances: une dualité source de questionnements éthiques. BioéthiqueOnline. 2016;5/26.

8. Beaudry J-S. The anxious heart of injustice: negative affective responses to disabilities. BioéthiqueOnline. 2016;5/28.

9. Doudenkova V. La bioéthique, l'art et le syndrome des ovaires polykystiques: propos impressionniste visant à réhabiliter les corps tabous et les ovaires blâmés. BioéthiqueOnline. 2016;5/22.

10. Noury M. Na-no-body: De l'oubli du corps sensible en nanomédecine. BioéthiqueOnline. $2016 ; 5 / 20$.

11. Kinkead A. 54 rue DuBalcon. BioéthiqueOnline. 2016;5/23.

12. Coleman S. Na-no-body. BioéthiqueOnline. 2016;5/19.

13. Stokes G. Constant Beauty Within and PCOS as Cacti. BioéthiqueOnline. 2016;5/21.

14. Le Mée M. Mael-e(s)t-France. BioéthiqueOnline. 2016;5/27.

15. Turcot K. «Médecine à deux vitesses à la manière de SUCCESs » ou "Deux poids deux mesures ou Quod licet lovi, non licet bovi c'est-à-dire "ce qui est permis à Jupiter ne l'est pas aux vaches" „ BioéthiqueOnline. 2016;5/25. 